\title{
Ecoulement et dissipation sur les déversoirs en gradins de gabions
}

\author{
L. Peyras, P. Royet, G. Degoutte*
}

\section{Introduction}

Les gabions demeurent des matériaux privilégiés dans la réalisation d'ouvrages hydrauliques. En Afrique Sahélienne notamment, le contexte social, économique et technique rend leur utilisation courante dans les petits barrages en terre. Ces retenues sont de conception relativement standardisée; en particulier, on emploie usuellement les gabions dans les évacuateurs de crue. A cet égard, les évacuateurs en gradins de gabions sont une solution fréquemment adoptée. Ces structures déversantes présentent une grande stabilité mécanique ainsi qu'une bonne résistance au déferlement de crues; en outre, leur mise en œuvre est aisée.

Cependant, les règles de dimensionnement de ce type de déversoirs sont mal établies, en particulier en ce qui concerne la méthode de calcul du bassin de dissipation. Dans l'état actuel des connaissances, les manuels traitant d'ouvrages en gabions proposent au mieux des méthodologies analogues à celles utilisées pour les déversoirs en pentes inclinées sans gradins [3], ou pour les déversoirs en chute verticale [1]. En aucun cas les calculs mentionnés ne prennent en compte la pré-dissipation qu'offre la structure en marches d'escalier, si bien que le bassin de dissipation est largement surdimensionné. Seul STEPHENSON a expérimenté des seuils en gradins de gabions à l'échelle 1/10 [9]. Cependant, les modèles testés n'excèdent pas $4 \mathrm{~m}$ de hauteur, et surtout, ils font intervenir des infiltrations à travers le parement amont, ce qui limite l'application de ses résultats aux seuils perméables en rivière.

Devant la complexité des écoulements sur les gradins et au sein des gabions, des méthodes de calcul telles que la modélisation numérique sont difficilement applicables et les modèles réduits restent l'outil privilégié. De nombreux déversoirs en gradins ont fait l'objet d'expérimentations. Elles concernent essentiellement les grands barrages en béton, où les phénomènes d'infiltrations sont exclus et où les régimes hydrauliques transitoires, fondamentaux dans notre étude, sont négligeables [4], [8].

Nous avons donc réalisé une expérimentation sur modèles réduits afin d'observer les écoulements sur des petits déversoirs en marches d'escaliers homogènes, de quantifier précisément la dissipation de l'énergie sur les déversoirs "standards" en gradins de gabions, et d'établir les paramètres qui détermineront le bassin de dissipation. Enfin, nous nous sommes attachés à analyser les déformations des gabions et les problèmes liés à la pérennité de l'ouvrage lors du passage des crues.

* CEMAGREF, groupement d'Aix-en-Provence, B.P. 31, Le Tholonet, 13612 Aix en Provence Cedex 1, Tél. 42669310

\section{Flows and dissipation of energy on gabion weirs}

The use of gabions in the small dams in the Sahel regions of Africa is quite common, they are used in flood spillways in which the gabion weirs are usual techniques. Indeed, apart from a good mechanical stability and a high level of resistance to flood flows, gabion weirs enable a large amount of predissipation of water energy before the stilling basin. In the present state of the technique the right dimensions for this type of structure are not well known. To define energy dissipation on the shoulders a systematic study of the standardized wasteways from 3 to $5 \mathrm{~m}$ high, for a flow rate of up to $3 \mathrm{~m}^{3} / \mathrm{s} \mathrm{per}$ meter width, has been carried out on scale models on 1/5 scale.

It has enabled the amount of energy dissipation to be quantified and enabled determination of dimensioning parameters for the stilling basin. The results obtained lead to a saving of 10 to $30 \%$ over the length of the stilling basin compared to the lengths obtained with the methods used up to present. Moreover, we also wished to classify the flows on the shoulders and define the phenomena observed during the different hydraulic regimes. 


\section{Expérimentation sur modèles réduits}

\section{Les modèles réduits}

L'échelle 1/5 adoptée est suffisamment grande pour se rapprocher au plus près des phénomènes hydrauliques réels et minimiser les erreurs de similitude et de mesure. Les gabions en modèle réduit sont rigoureusement à l'échelle 1/5: dimensions géométriques $(20 \mathrm{~cm}$ $\times 20 \mathrm{~cm} \times 60 \mathrm{~cm}$ soit des gabions réels de $1 \mathrm{~m} \times 1 \mathrm{~m} \times 3 \mathrm{~m})$, mailles hexagonales torsadées $(20 \mathrm{~mm} \times 30 \mathrm{~mm}$ soit une maille réelle du type $100 \times 120)$, diamètre des fils $(0,7 \mathrm{~mm}$ soit un diamètre réel de $3,5 \mathrm{~mm}$ ), et granulats de remplissage (ballast de 30 à $40 \mathrm{~mm}$ ). La terminologie et les unités utilisées sont décrites en annexe.

L'expérimentation a été conduite dans le canal vitré de la Société du Canal de Provence. Sa largeur est de $80 \mathrm{~cm}$. Les débits simulés s'étendent de 0,5 à $3 \mathrm{~m}^{3} \cdot \mathrm{s}^{-1} \cdot \mathrm{ml}^{-1}$ à raison d'une dizaine de débits par expérience. Seul le parement aval de barrage est reproduit; le parement amont est remplacé par une membrane étanche.

Nous avons testé la dissipation sur des déversoirs dont les caractéristiques prennent les valeurs suivantes (fig. 1):

- pente du parement aval : $1 / 1,1 / 2$ et $1 / 3$;

- hauteur du déversoir : $3 \mathrm{~m}, 4 \mathrm{~m}$ et $5 \mathrm{~m}$, soit respectivement 3,4 et 5 gradins, la hauteur d'un gradin étant standardisée $(1 \mathrm{~m})$.

Différents profils de gradins ont été reproduits (fig. 2):

— gradins en gabions ou gabions " nus";

- gradins dont les girons sont protégés par une galette horizontale en béton; la technique de la "galette en béton " est préconisée quand les crues sont accompagnées de transport solide ;

- gradins dont les girons sont protégés par une galette en béton en "contre-pente" ;

- gradins équipés à leur nez d'un "contre-seuil " en gabion semelle.

Sur les déversoirs de surface, les écoulements d'eau sont caractérisés par des vitesses rapides. La force gravitaire est prépondérante sur les autres forces extérieures et on adopte la similitude de Froude. Tous les résultats seront donnés à l'échelle réelle.

\section{L'instrumentation}

Les charges spécifiques en pied de déversoir sont mesurées à l'aide d'une rampe de Pitot, c'est-à-dire d'une série de tubes de Pitot répartis tous le long d'une section transversale et couplés à un même tube de lecture. Cette rampe, réalisée au moyen d'un tube de cuivre percé de petits orifices ouverts face aux lignes de courant, intègre la charge spécifique $\left(H_{s}\right)$ sur une section du déversoir. Contrairement à un tube de Pitot qui enregistrerait toutes les fluctuations de l'écoulement en un point précis de la section, la rampe de Pitot offre une lecture stable de $H_{s}$. Un étalonnage précis de la rampe a donné entre 6 et $10 \%$ d'erreur par défaut selon le débit; les résultats expérimentaux ont été alors majorés en conséquence.

Le débit alimentant le canal vitré est mesuré par un déversoir triangulaire normalisé à mince paroi. Des jaugeages volumétriques ont montré moins de $3 \%$ d'erreur sur le débit.

\section{Méthodologie de l'expérimentation}

La charge initiale totale de l'écoulement au-dessus du seuil $\left(E_{0}\right)$ est calculée dans la section critique à partir du débit :

$$
E_{0}=H+3 / 2 Y_{c}
$$

avec

$H$ : hauteur du déversoir

$Y_{c}$ : tirant d'eau critique ; $Y_{c}=\left(q^{2} / 2 g\right)^{1 / 3}, q$ étant le débit par unité de longueur de seuil en $\mathrm{m}^{3} \cdot \mathrm{s}^{-1} \cdot \mathrm{ml}^{-1}$

La charge résiduelle $\left(E_{1}\right)$ au pied du déversoir (à l'entrée du bassin de dissipation en amont du ressaut) est mesurée au moyen de la rampe de Pitot. On calcule alors la perte de charge $E_{0}-E_{1}$.

\section{Ecoulements sur les gradins}

L'expérimentation a mis en évidence deux types d'écoulements: les écoulements en nappe et les écoulements très turbulents.

\section{Les écoulements en nappe (nappe flow)}

Les écoulements en nappe sont observés pour les petits à moyens débits. La lame d'eau déversante frappe le giron de la marche inférieure, d'abord totalement puis partiellement, et on parle alors respectivement de nappe isolée et de nappe partielle.

\section{Ecoulements en nappe isolée}

Deux régimes hydrauliques sont alors possibles: un premier caractérisé par une alternance des régimes fluvial et torrentiel, et un second totalement torrentiel.

a) Alternance des régimes fluvial et torrentiel: un ressaut hydraulique s'amorce en aval immédiat de l'impact de la lame déversante. Cet écoulement n'a été observé qu'avec' une contre-pente ou un contre-seuil et pour de faibles débits (inférieurs à $1 \mathrm{~m}^{3} \cdot \mathrm{s}^{-1} \cdot \mathrm{ml}^{-1}$ ).

Une modélisation simple peut être proposée dans ce seul cas et donne de bons résultats en regard de nos expériences (fig. 3) :

- au point d'impact de la lame déversante, l'écoulement prend un tirant d'eau minimal $Y_{1}$. Sous la lame déversante au pied de la marche apparaît un coussin d'eau de tirant d'eau $Y_{p}$;

- le ressaut s'amorce dans la section 1;

- en aval immédiat du ressaut dans la section 2, l'écoulement devient fluvial et prend le tirant d'eau $Y_{2}$

- entre la section 2 et la section 3, l'écoulement acquiert de la vitesse du fait de la chute et redevient torrentiel avec passage par le tirant d'eau critique $Y_{c}$ dans la section 3 .

Les conditions hydrauliques sont identiques d'un gradin à un autre; l'écoulement dissipe alors, à chaque marche, une énergie équivalente à une hauteur de 
sens du courant

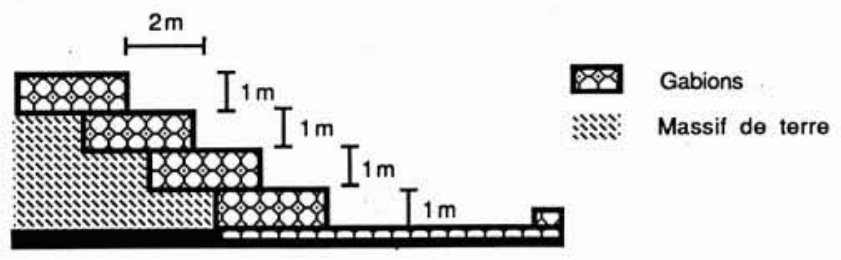

1. Exemple de parement aval en gradins de gabions, de $4 \mathrm{~m}$ de hauteur et de pente 1/2; au pied du déversoir, le bassin de dissipation gabionné.

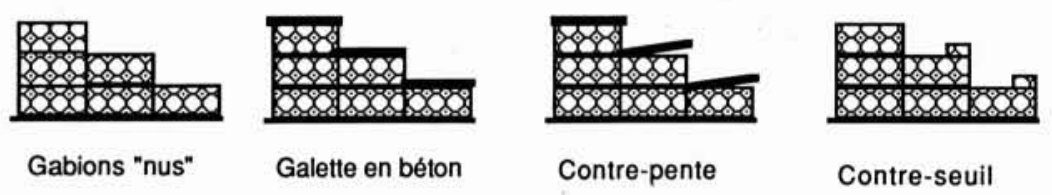

2. Les différents profils de gradins testés.

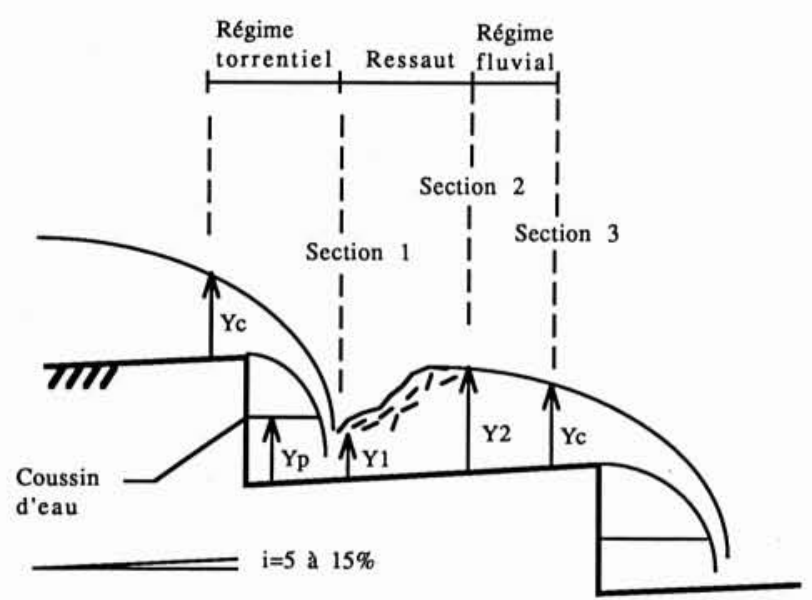

3. Ecoulement en nappe isolée avec alternance de régimes fluvial et torrentiel.

marche. Par suite, on peut proposer une formule de perte d'énergie sur les déversoirs en escalier et pour des écoulements en nappe isolée :

- énergie sur la crête du déversoir :

$E_{0}=n \cdot h+3 / 2 Y_{c}$, avec un déversoir de $n$ marches de $h$ mètres de hauteur chacune $(n h=H)$.

- énergie au pied du déversoir :

$$
\begin{gathered}
E_{1}=Y_{1}+V_{1}^{2} / 2 g \\
\Rightarrow E_{0}-E_{1}=n \cdot h+3 / 2 Y_{c}-Y_{1}-q^{2} /\left(2 g \cdot Y_{1}^{2}\right)
\end{gathered}
$$

RAND a étudié l'hydraulique d'une chute d'eau sur une marche bétonnée de $h$ mètres de hauteur; il propose un système d'équations empiriques définissant les différents paramètres : [7]

$$
\begin{aligned}
& Y_{1} / h=0,54 \cdot\left(q^{2} /\left(g \cdot h^{3}\right)\right)^{0,425} \\
& Y_{2} / h=1,66 \cdot\left(q^{2} /\left(g \cdot h^{3}\right)\right)^{0,27} \\
& Y_{p} / h=\left(q^{2} /\left(g \cdot h^{3}\right)\right)^{0,22}
\end{aligned}
$$

$q$ étant le débit en $\mathrm{m}^{3} \cdot \mathrm{s}^{-1} \cdot \mathrm{ml}^{-1}$ de la lame d'eau et $\left(Y_{1}, Y_{2}, Y_{p}\right)$ les tirants d'eau définis ci-dessus. 


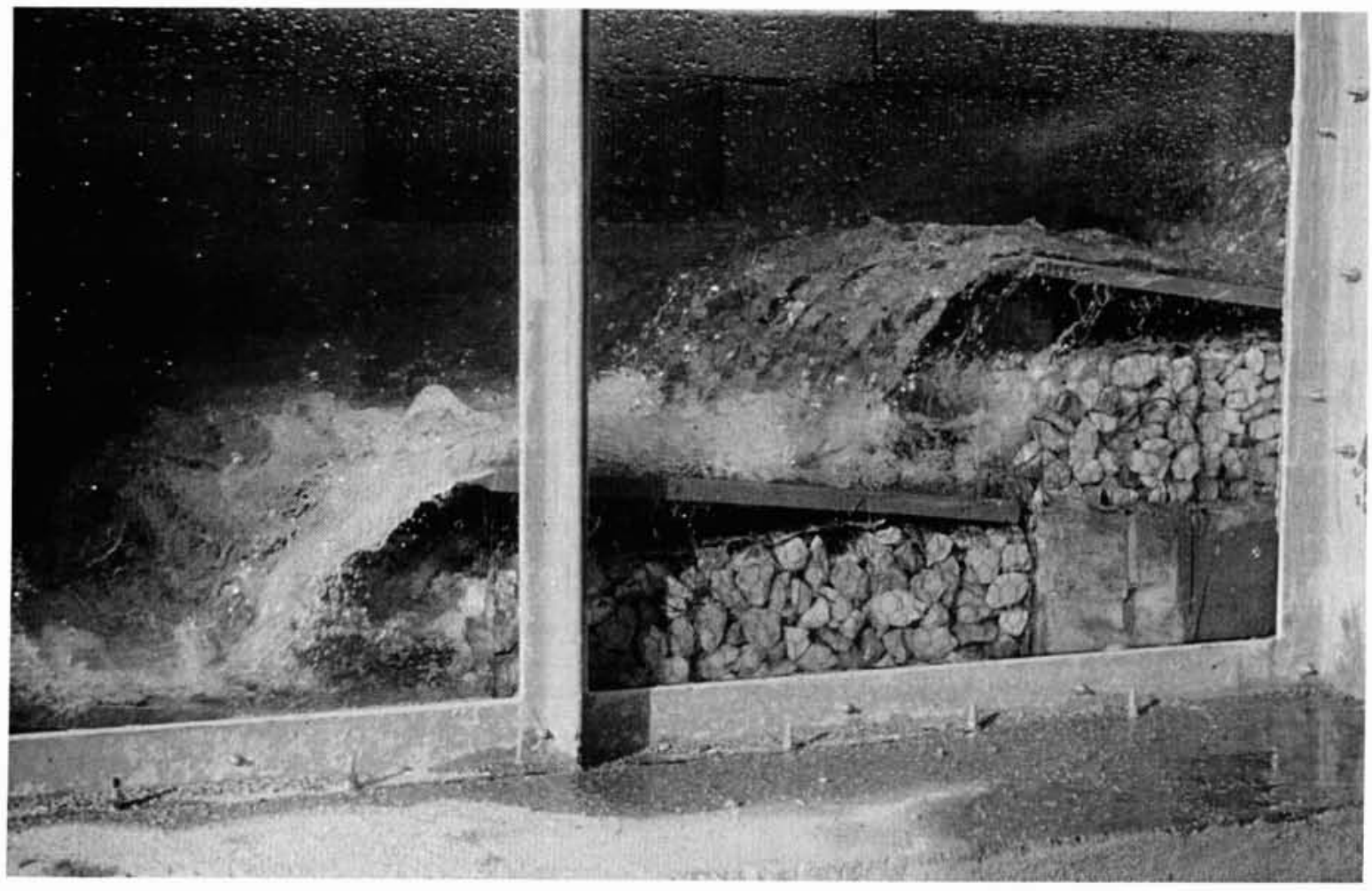

Ecoulement en nappe isolée sur un déversoir de pente $1 / 3$ et de $4 \mathrm{~m}$ de hauteur ; gradins équipés d'une contre-pente; alternance des régimes fluvial et torrentiel ; débit $0,60 \mathrm{~m}^{3} \cdot \mathrm{s}^{-1} \cdot \mathrm{ml}^{-1}$.

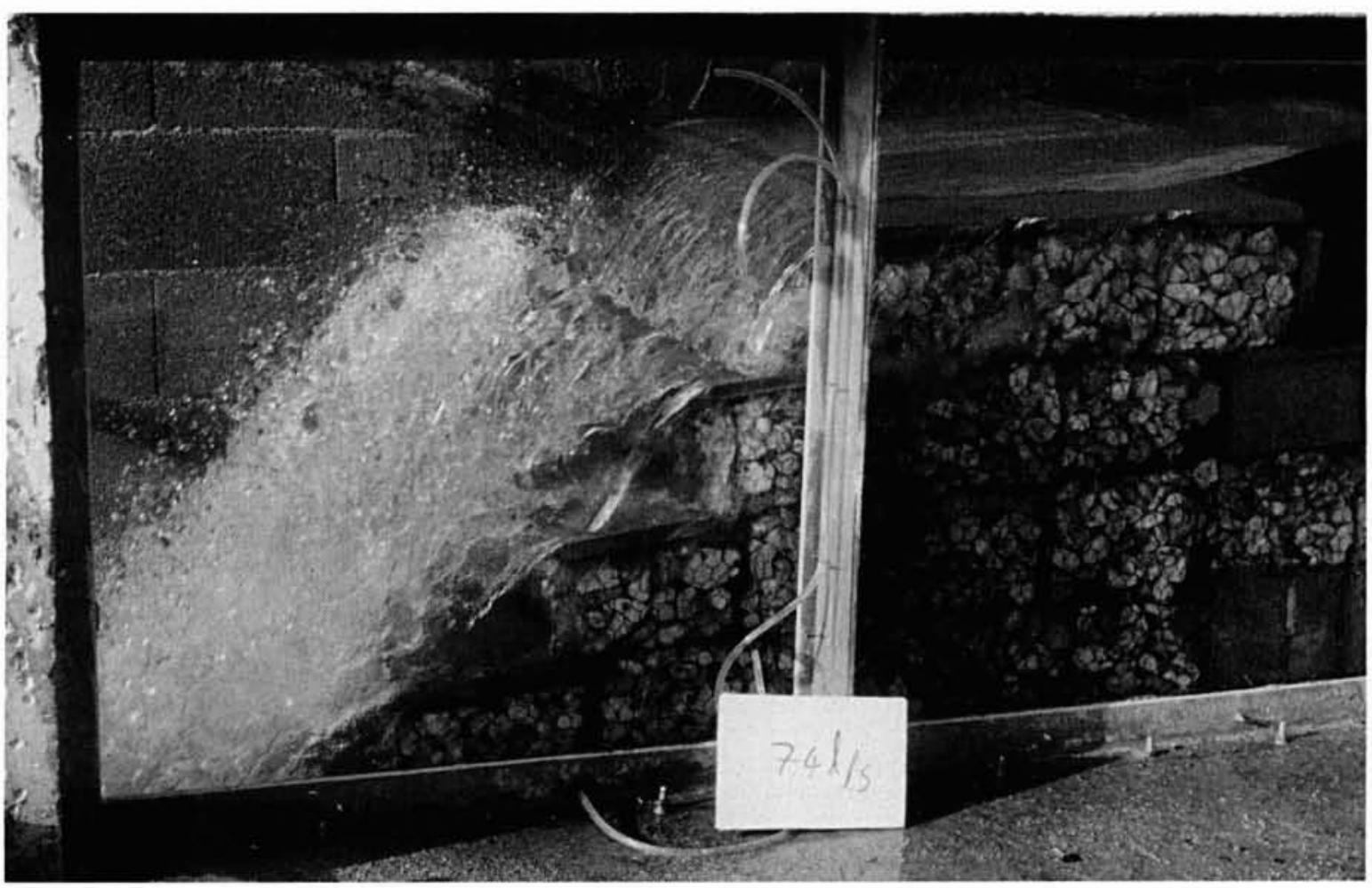

Ecoulement en nappe isolée sur un déversoir de pente $1 / 1$ et de $5 \mathrm{~m}$ de hauteur; gradins protégés par une galette en béton: régime torrentiel; débit $1,03 \mathrm{~m}^{3} \cdot \mathrm{s}^{-1} \cdot \mathrm{ml}^{-1}$. 


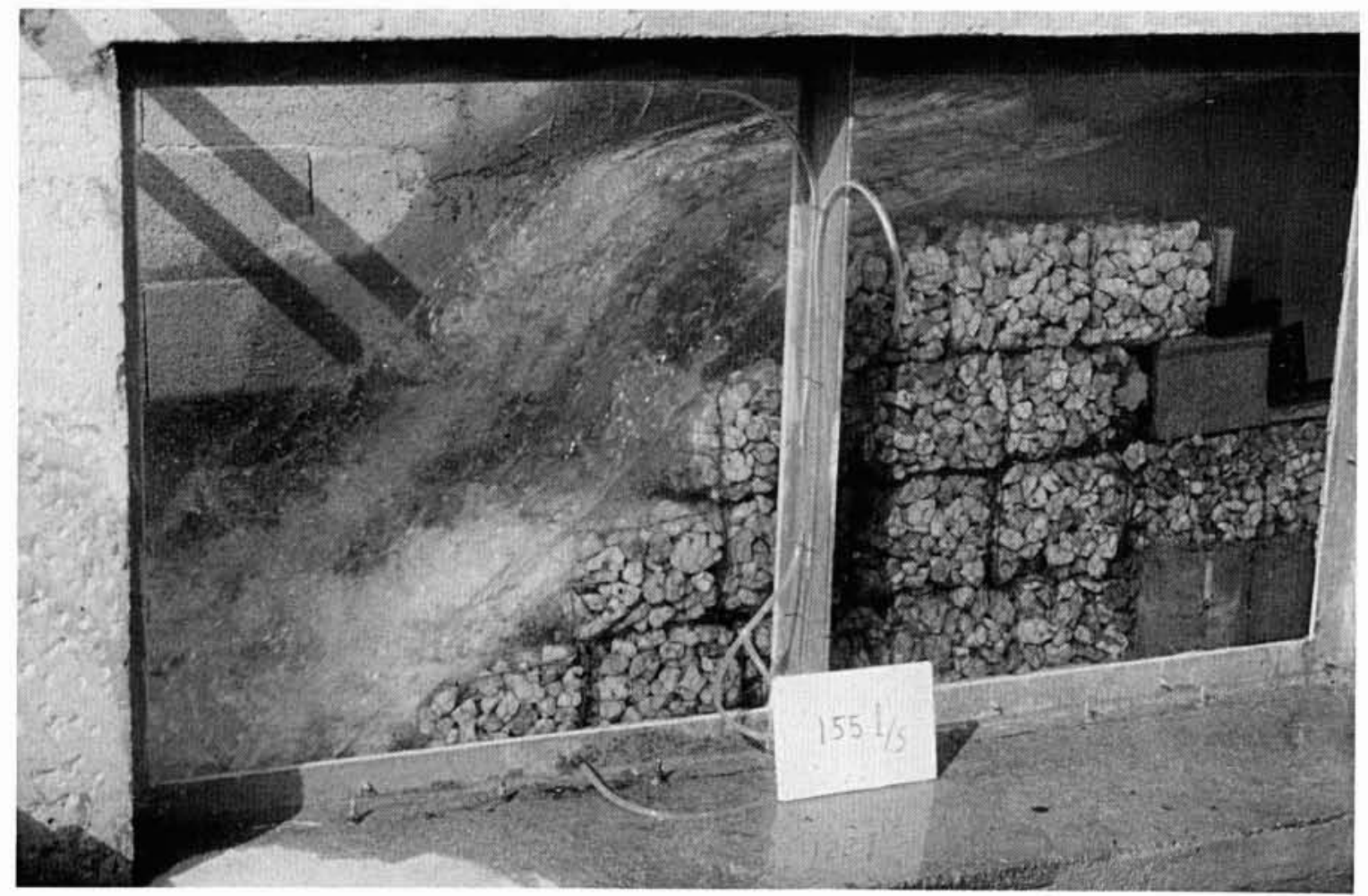

Ecoulement très turbulent sur un déversoir de pente $1 / 1$ et de $5 \mathrm{~m}$ de hauteur : gradins en gabions " nus "; régime torrentiel : débit $2,17 \mathrm{~m}^{3} \cdot \mathrm{s}^{-1} \cdot \mathrm{ml}^{-1}$.

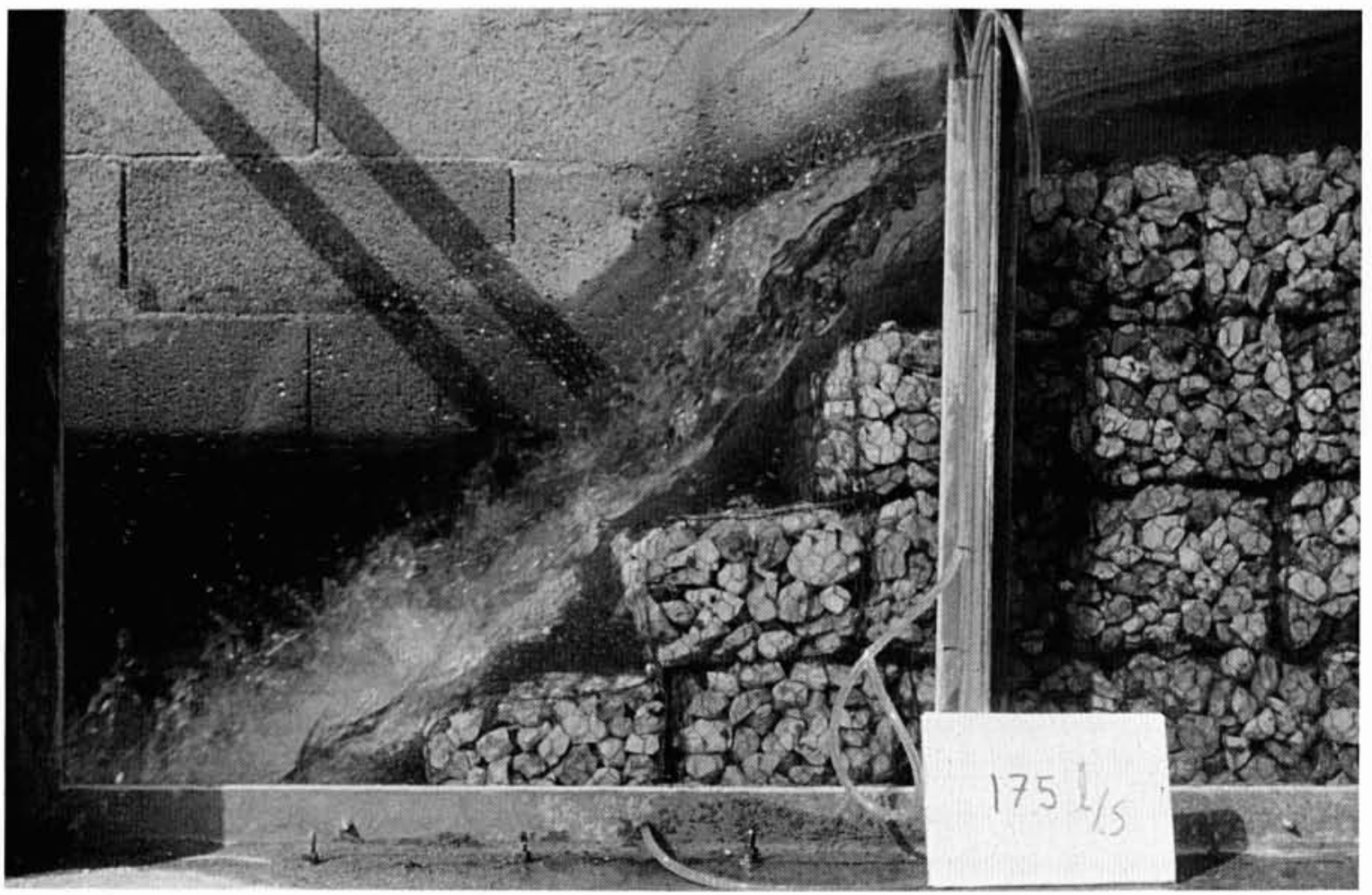

Ecoulement très turbulent sur un déversoir de pente $1 / 1$ et de $5 \mathrm{~m}$ de hauteur ; gradins en gabions " nus "; régime torrentiel ; débit $2,45 \mathrm{~m}^{3} \cdot \mathrm{s}^{-1} \cdot \mathrm{ml}^{-1}$. 


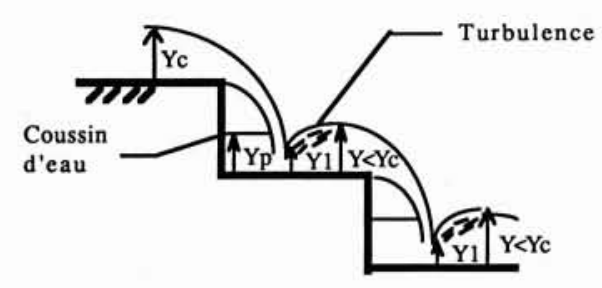

4. Ecoulement en nappe isolée avec régime constamment torrentiel.

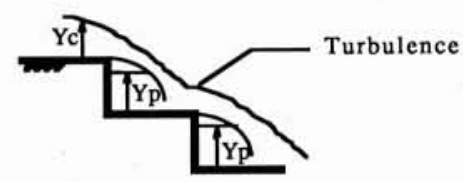

5. Ecoulement en nappe partielle.

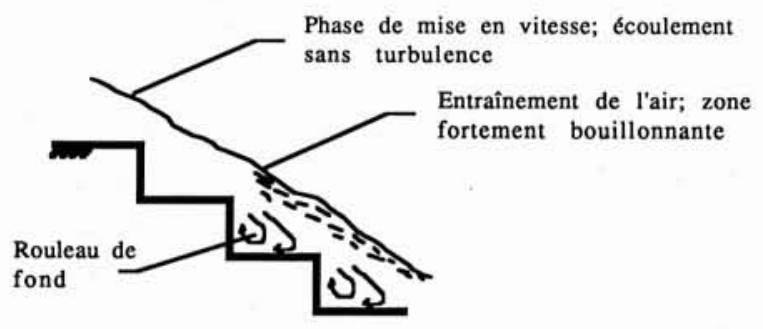

6. Ecoulement très turbulent.

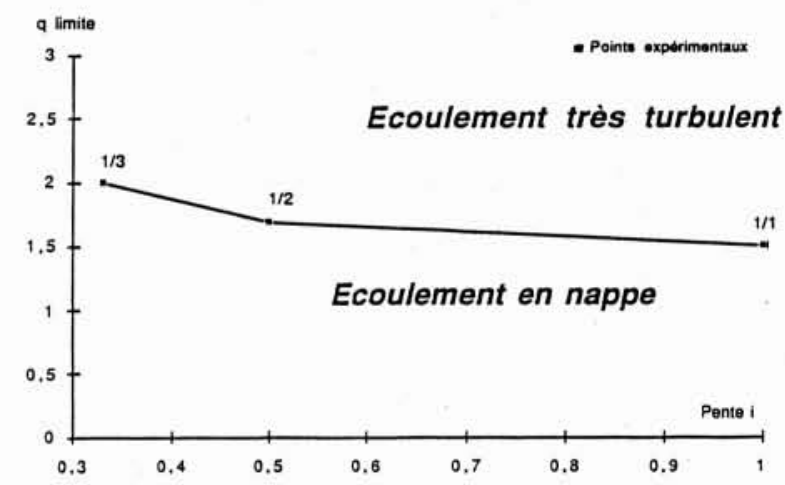

7. Débit unitaire limite $q\left(\mathrm{~m}^{3} / \mathrm{s} / \mathrm{ml}\right)$ entre les écoulements en nappe et les écoulements très turbulents, en fonction de la pente $i(\mathrm{~m} / \mathrm{m})$ pour des déversoirs en gradins de gabions "nus".
La combinaison des équations (1) et (2) permet de calculer la perte de charge sur les déversoirs en escalier et on peut vérifier la validité de ces formules pour des structures en gradins de gabions en prenant $h=1 \mathrm{~m}$. Les résultats de nos expériences, concernant les écoulements en nappe isolée mais aussi l'ensemble des écoulements en nappe, sont proches des résultats obtenus avec la modélisation: la dissipation réelle est au maximum $10 \%$ supérieure à la dissipation calculée à partir de la modélisation. Cette différence entre l'expérimentation et la modélisation s'explique car certains paramètres comme les infiltrations dans les gabions, les différences de rugosité entre le gabion et le béton ou encore la pente du déversoir, ne sont pas considérés dans la modélisation.

b) Régime torrentiel: En absence de contre-seuil ou de contre-pente, la vitesse de l'eau reste forte et le ressaut hydraulique est chassé en aval de la marche. On observe un fort bouillonnement en aval de la zone d'impact de la lame déversante, mais l'écoulement reste torrentiel tout le long du déversoir (fig. 4).

Les écoulements en nappe sur les gradins horizontaux (profils "gabions nus" et "galette en béton") sont systématiquement en régime torrentiel. Sur les déversoirs équipés d'une contre-pente ou d'un contre-seuil, le régime devient torrentiel à partir d'un certain débit.

\section{Ecoulements en nappe partielle}

La lame déversante frappe pour partie le giron de la marche inférieure et l'éclatement des jets à chaque gradin provoque un fort bouillonnement. Le régime reste torrentiel tout le long des gradins (fig. 5).

\section{Dissipation des écoulements en nappe}

La dissipation de l'énergie des écoulements en nappe se réalise en deux phases: lors du choc de la lame sur la marche, puis essentiellement dans la zone de bouillonnement qui suit l'éclatement du jet, avec ou sans formation d'un ressaut.

On peut évaluer dans une première approche la dissipation de l'énergie sur les déversoirs en gradins de gabions en extrapolant la modélisation des écoulements en nappe isolée à l'ensemble des écoulements en nappe. Nos résultats ont montré que cette extrapolation à partir des formules (1) et (2) est valide à $10 \%$ près.

\section{Les écoulements très turbulents (skimming flow)}

Ils sont observés pour les moyens à forts débits; on ne peut plus distinguer alors de lame d'eau et le déversoir est totalement immergé dans un courant fort et relativement lissé.

D'amont en aval, on peut distinguer deux zones (fig. 6) :

- une première zone transitoire sur les deux premiers gradins: l'écoulement s'accélère jusqu'à atteindre une vitesse limite maximale $V_{\max }$ où les phénomènes d'entraînement de l'air apparaissent; l'écoulement ne présente pas de bouillonnement dans cette phase de mise en vitesse et la surface libre du courant forme une ondulation lisse au-dessus du déversoir ;

- l'écoulement a atteint la vitesse $V_{\max }$ d'apparition de l'entraînement de l'air. Les particules d'air se mêlent 
alors au courant d'eau et un bouillonnement intense se prolonge en s'amplifiant jusqu'au pied du déversoir. Le coussin d'eau au pied des gradins a laissé place à un rouleau de fond dans lequel on aperçoit la rotation de bulles d'air entraînées ; le courant se déplace le long du déversoir, s'appuyant sur le rouleau de fond d'une part et sur le nez des marches d'autre part.

La vitesse d'apparition de l'entraînement de l'air ne dépend que des caractéristiques du déversoir, en particulier de la hauteur des marches (ici $h=1 \mathrm{~m}$ ) et de la longueur des girons. Quel que soit le débit de la crue simulée (jusqu’à $3 \mathrm{~m}^{3} \cdot \mathrm{s}^{-1} \cdot \mathrm{ml}^{-1}$ ), nous avons mesuré $V_{\max }$ de l'ordre de 5,5 à $6 \mathrm{~m} / \mathrm{s}$ selon la pente du déversoir. Une fois $V_{\max }$ atteint, on note une légère diminution de la vitesse de l'eau; outre une perte de $h$ mètres d'énergie potentielle par gradin, l'écoulement dissipe aussi une partie de son énergie cinétique.

RAJARATNAM propose une modélisation des écoulements très turbulents sur la base d'expérimentations menées par SORENSEN [6], [8]

\section{Limites entre écoulements en nappe et écoulements très turbulents}

Les écoulements très turbulents apparaissent pour des débits plus élevés que les écoulements en nappe. Bien entendu, les caractéristiques géométriques des gradins interviennent dans l'initialisation de l'entraînement de l'air: une pente forte et des gradins horizontaux favorisent les écoulements très turbulents. La figure 7 présente les limites entre les deux régimes d'écoulements.

\section{Dissipation de l'énergie sur les déversoirs en gradins de gabions}

\section{Présentation adimensionnelle des résultats}

Les paramètres régissant les écoulements sur les gradins de gabions sont :

- la pente $i$ et la hauteur $H$ du déversoir, qui définissent totalement la géométrie du parement aval ( $H$ mètres équivalent à $H$ gradins);

- le profil du gradin, avec la présence ou non de contreseuil ou de contre-pente; dans un premier temps, nous nous limiterons aux gradins de gabions " nus " avec des girons horizontaux.

- le débit par unité de longueur, qui régit, avec la pente $i$, le type d'écoulement (en nappe ou très turbulent).

- la force gravitaire prépondérante sur toutes les forces extérieures.

Quatre paramètres sont donc nécessaires, dans le cas des gradins de gabions " nus ", pour exprimer complètement la variable adimensionnelle $\left(E_{0}-E_{1}\right) / H$ (perte de charge par unité de hauteur du déversoir):

$$
\left(H_{0}-E_{1}\right) / H=f(q, H, i, g)
$$

On peut déterminer classiquement deux paramètres adimensionnels décrivant $\left(E_{0}-E_{1}\right) / H$ :

- le premier tout logiquement choisi est la pente de l'escalier : $i$

- le second fait intervenir $q, H$ et $g$ de façon comparable au carré du nombre de Froude :

$$
q^{2} /\left(g \cdot H^{3}\right) .
$$

Ainsi, on peut écrire :

$$
\left(E_{0}-E_{1}\right) / H=f_{1}\left(i, q^{2} /\left(g \cdot H^{3}\right)\right) .
$$

De la même façon, la variable adimensionnelle $Y_{1} / H$ (tirant d'eau au pied du déversoir sur la hauteur du même déversoir) s'exprime :

$$
Y_{1} / H=f_{2}\left(i, q^{2} /\left(g \cdot H^{3}\right)\right) .
$$

A partir des résultats expérimentaux, nous avons tracé des abaques (fig. 8 et 9 , page suiv.) donnant en ordonnée $Y_{1}=\left(E_{0}-E_{1}\right) / H$ et $Y_{2}=Y_{1} / H$ en fonction de l'abscisse $X=q^{2} /\left(g \cdot H^{3}\right)$ et du paramètre $i$ de la façon suivante : - compte tenu que $Y_{1}$ et $Y_{2}$ tendent respectivement vers 1 et 0 lorsque $X$ tend vers zéro, les courbes retenues sont de la forme

$$
1 /\left(1-Y_{1}\right)=a_{1} \cdot X^{b_{1}} \text { et } Y_{2}=a_{2} \cdot X^{b_{2}} \text {; }
$$

- on ajuste ces courbes aux nuages de points par la méthode des moindres carrés après transformation logarithmique.

Les coefficients ajustés sont donnés sur les tables I et II (page suiv.).

\section{Domaine de validité des abaques}

On se limite aux débits inférieurs à $3 \mathrm{~m}^{3} \cdot \mathrm{s}^{-1} \cdot \mathrm{ml}^{-1}$. Audelà, des risques d'endommagements du déversoir sont à craindre (cf. section "déformations des gabions »).

Les abaques (fig. 8 et 9) prennent en compte les expériences conduites sur des déversoirs de 3,4 et $5 \mathrm{~m}$ de hauteur. La représentation adimensionnelle permet d'extrapoler ces résultats. Nous préconisons néanmoins de se limiter à des hauteurs comprises entre 2 et $7 \mathrm{~m}$ car, pour des hauteurs plus importantes, on s'éloignerait par trop du domaine de nos expériences.

Bien entendu, on peut interpoler les résultats pour les déversoirs de pentes intermédiaires, comme par exemple $1 / 1,5$ ou $1 / 2,5$.

\section{Méthodologie de dimensionnement du bassin de dissipation}

Connaissant la géométrie du déversoir (pente, hauteur et longueur déversante) et la crue de projet, nos travaux permettent de déterminer le tirant d'eau $Y_{1}$ correspondant à des gradins de gabions "nus" (abaque fig. 9).

Pour dimensionner le bassin de dissipation, on calcule alors (fig. 10):

- le nombre de Froude $F_{1}$ au pied du déversoir :

$$
F_{1}=q\left(g \cdot Y_{1}^{3}\right)^{1 / 2}
$$




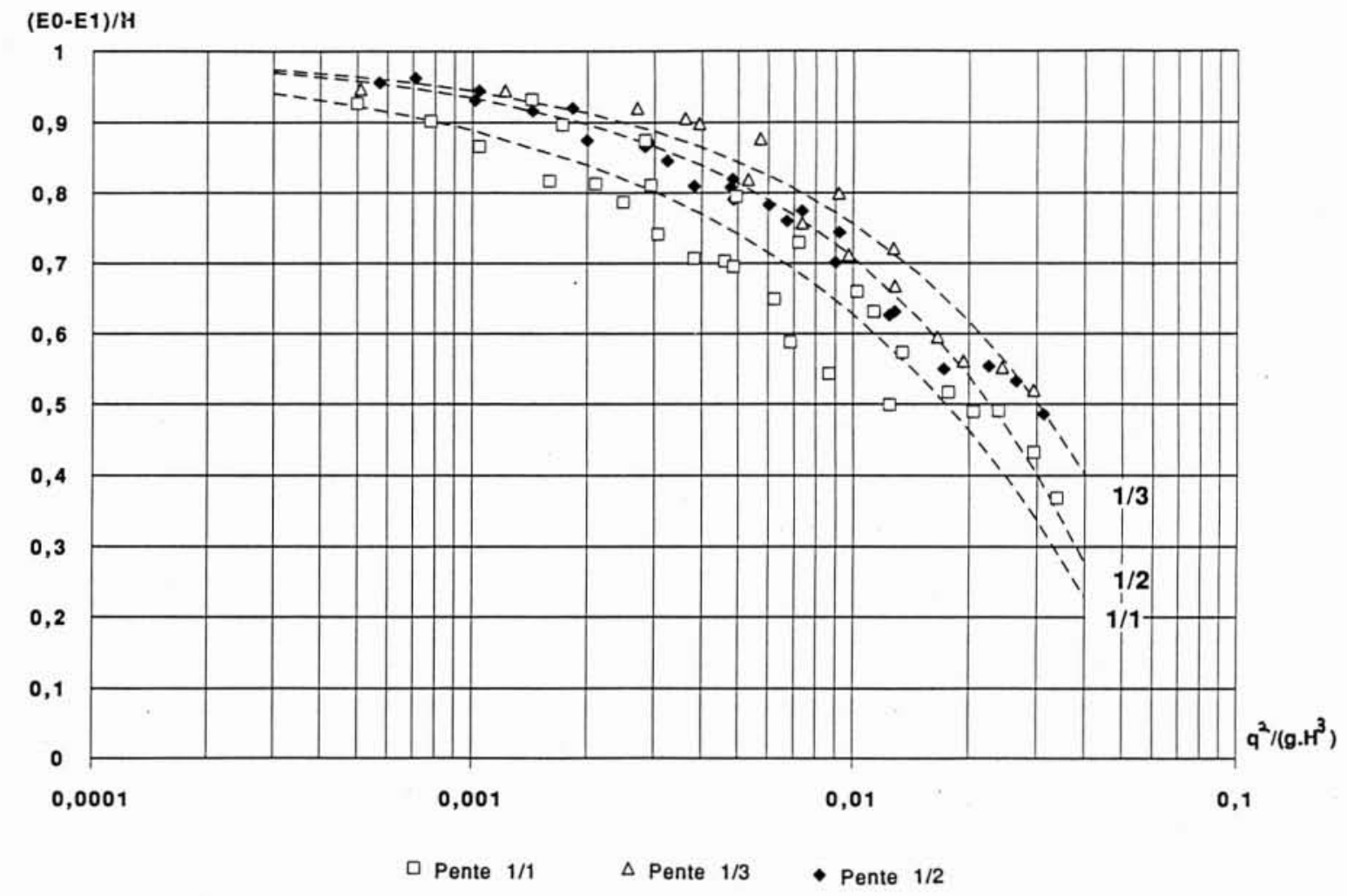

8. Perte de charge unitaire sur un déversoir en gradins de gabions "nus".

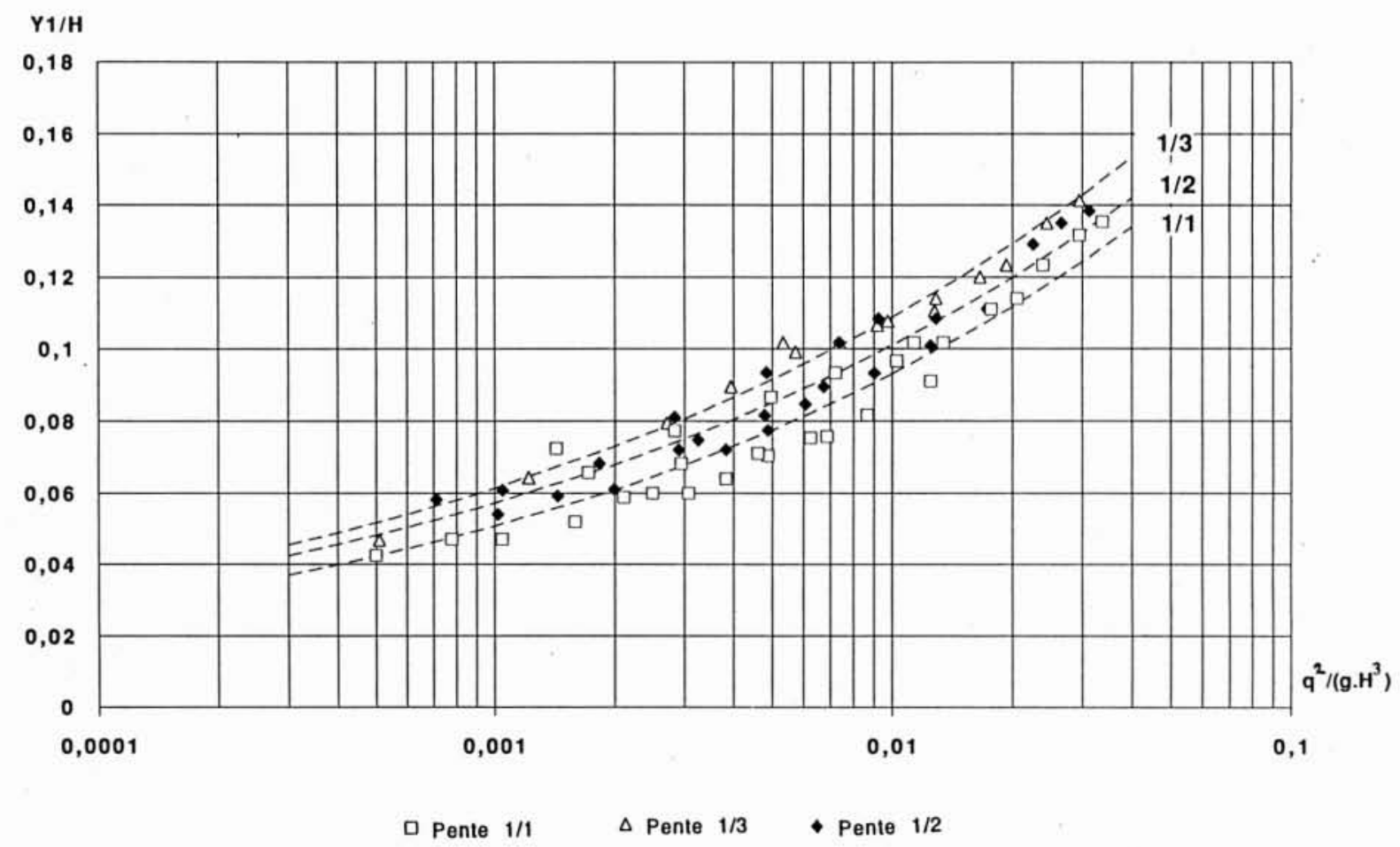

9. Tirant d'eau $Y_{1}$ au pied d'un déversoir en gradins de gabions

"nus". 


\section{Table I}

Relation entre $Y_{1}=\left(E_{0}-E_{1}\right) / H$ et $X=$ $q^{2} /\left(g . H^{3}\right)$ sous la forme $1 /\left(1-Y_{1}\right)=a_{1} \cdot X^{b_{1}}$; coefficients $a_{1}$ et $b_{1}$ ajustés; coefficient de détermination $r^{2}$.

\begin{tabular}{|cc|c|c|c|}
\cline { 3 - 5 } \multicolumn{1}{c|}{} & Ln $\left(\mathrm{a}_{1}\right)$ & $\mathrm{b}_{1}$ & $\mathrm{r}^{2}$ (Coef. détermination) \\
\hline Pente $1 / 3$ & $(16$ couples $)$ & $-1,568$ & $-0,647$ & 0,904 \\
\hline Pente $1 / 2$ & $(25$ couples $)$ & $-1,778$ & $-0,654$ & 0,964 \\
\hline Pente $1 / 1$ & $(28$ couples $)$ & $-1,434$ & $-0,526$ & 0,874 \\
\hline
\end{tabular}

\section{Table II}

Relation entre $Y_{2}=Y_{1} / H$ et $X=q^{2} /\left(g \cdot H^{3}\right)$ sous la forme $Y_{2}=a_{2} \cdot X^{b_{2}}$; coefficients $a_{2}$ et $b_{2}$ ajustés; coefficient de détermination $r^{2}$.

\begin{tabular}{|cc|c|c|c|}
\cline { 3 - 5 } \multicolumn{1}{c|}{} & Ln (a2) & $\mathrm{b}_{2}$ & $\mathrm{r}^{2}$ (Coef. détermination) \\
\hline Pente $1 / 3$ & $(15$ couples) & $-1,074$ & 0,248 & 0,974 \\
\hline Pente $1 / 2$ & $(24$ couples $)$ & $-1,157$ & 0,247 & 0,941 \\
\hline Pente $1 / 1$ & $(28$ couples $)$ & $-1,163$ & 0,263 & 0,888 \\
\hline
\end{tabular}

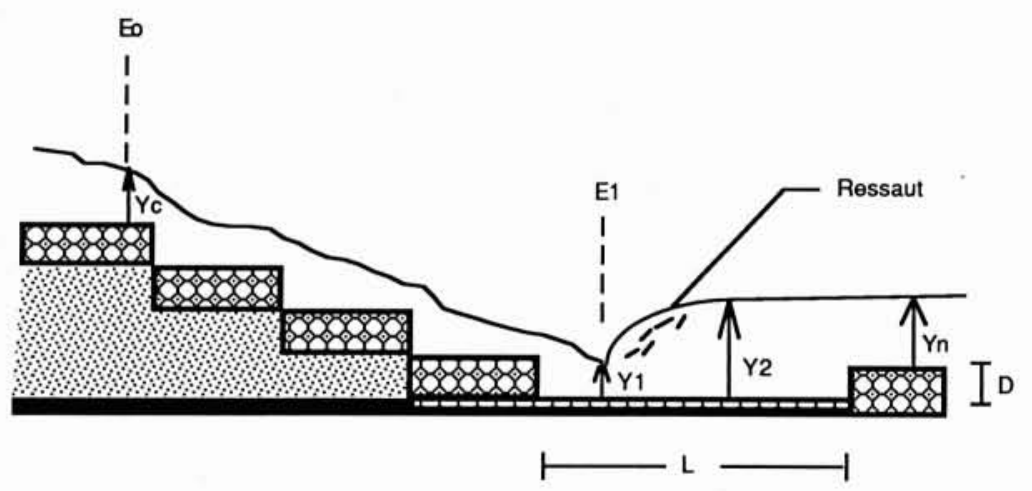

10. Dimensionnement du bassin de dissipation. 
- le tirant d'eau conjugué $Y_{2}$ en fin de ressaut:

$$
Y_{2}=0,5 \cdot Y_{1} \cdot\left[\left(1+8 \cdot F_{1}^{2}\right)^{1 / 2}-1\right]
$$

- la longueur $L$ du bassin de dissipation par la formule empirique [11]: $L=6 Y_{2}$

- la profondeur $D$ du bassin de dissipation permettant d'atteindre le tirant d'eau normal $Y_{n}$ de la rivière; $Y_{n}$ ne dépend que des conditions aval:

$$
D=Y_{n}-Y_{1} .
$$

Le type de gradin modifie la dissipation de l'énergie : - les galettes en béton coulées sur le giron des marches étanchent partiellement le déversoir et la dissipation est moindre; pour dimensionner alors exactement la fosse de dissipation, l'expérience montre que la longueur $L$, calculée par la méthode ci-dessus décrite correspondant aux gradins en gabions "nus", doit être majorée respectivement de $15 \%, 8 \%$ et $0 \%$ pour les pentes $1 / 3$, $1 / 2$ et $1 / 1$.

- les contre-pentes et les contre-seuils créent des poches d'eau qui amortissent les lames déversantes et induisent la formation de ressauts hydrauliques; la dissipation de l'énergie est donc améliorée. Selon la pente du déversoir, le bassin de dissipation est susceptible d'être raccourci jusqu'à $10 \%$ [5].

\section{Discussion}

La perte de charge par unité de hauteur $\left(E_{0}-E_{1}\right) / H$ décroît fortement lorsque $q^{2} /\left(g \cdot H^{3}\right)$ croît et donc, à hauteur $H$ constante, lorsque le débit augmente. Ce phénomène s'explique par le changement de régime (passage de l'écoulement en nappe à l'écoulement très turbulent $)$ qui se produit quand $q^{2} /\left(g . H^{3}\right)$ augmente. La dissipation de l'énergie des écoulements très turbulents est nettement inférieure à celle qui se réalise lors des écoulements en nappe.

On note également que les courbes des variables $\left(E_{0}-E_{1}\right) / H$ et $Y_{1} / H$ correspondant aux différentes pentes ont tendance respectivement à se confondre lorsque $q^{2} /\left(g . H^{3}\right)$, et donc $q$, diminue. En effet comme il est décrit dans la modélisation des écoulements en nappe isolée, on retrouve alors les mêmes conditions hydrauliques d'un gradin à un autre, et ce indépendamment de la pente du déversoir.

\section{Déformations des gabions}

Les expériences sur modèles réduits ont mis en évidence certaines déformations des gabions constituant le déversoir. Ces derniers sont sensibles au déplacement de leurs matériaux de remplissage. Il est primordial d'apporter un soin particulier à leur réalisation, ainsi qu'il est décrit dans [2]:

- qualité et agencement des pierres à la partie supérieure de ces gabions;

- strict respect des règles de granulométrie (dimension des matériaux supérieure à 1,5 fois la maille).

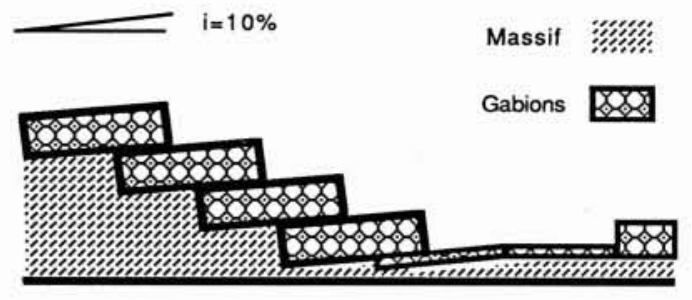

11. Déversoir en contre-pente.

Si les gabions sont exposés à de fortes crues (supérieures à $1,5 \mathrm{~m}^{3} \cdot \mathrm{s}^{-1} \cdot \mathrm{ml}^{-1}$ ), on renforce alors les grillages et les ligatures. On conseille également de rigidifier la cage métallique des gabions en disposant une troisième rangée de tirants supplémentaires et en augmentant le nombre de diaphragmes dans les gabions.

Les gabions sont sensibles au transport de matériaux solides, qui risquent de provoquer l'abrasion voire même la rupture du grillage. Le cas échéant, on protège le giron des gradins en coulant une galette en béton de 5 à $10 \mathrm{~cm}$.

On peut donner une contre-pente aux gabions, tout simplement en inclinant vers l'amont l'ensemble du déversoir (fig. 11). Les girons des marches peuvent dans ce cas également être protégés par une galette en béton.

Outre une excellente dissipation de l'énergie, cette structure augmente la stabilité de l'ouvrage. Elle requiert néanmoins une plus grande technicité pour la mise en œuvre des gabions inclinés.

\section{Conclusion}

Si toutefois les règles de mise en œuvre des gabions sont respectées, les déversoirs à parement aval en gradins de gabions peuvent supporter sans préjudice notable des crues jusqu'à $3 \mathrm{~m}^{3} \cdot \mathrm{s}^{-1} \cdot \mathrm{ml}^{-1}$. C'est indubitablement la seule structure déversante gabionnée capable de supporter de telles crues; en revanche, les parements aval en pentes inclinées gabionnés n'admettent pas des débits supérieurs à $1 \mathrm{~m}^{3} \cdot \mathrm{s}^{-1} \cdot \mathrm{ml}^{-1}[2],[5]$.

D'autre part, les déversoirs en gradins offrent une prédissipation de l'énergie importante avant le bassin de dissipation. Cette étude permet de quantifier précisément cette dissipation sur les gradins et les paramètres qui définissent le bassin.

Les résultats finaux conduisent à une économie de 10 à $30 \%$ sur la longueur du bassin de dissipation par rapport aux longueurs obtenues avec les méthodologies jusqu'à présent utilisées. Connaissant le coût de l'évacuateur de crue sur ce type de barrage, l'intérêt économique se situe entre 5 et $10 \%$ sur l'ensemble du projet.

\section{Remerciements}

Nous tenons à remercier la Société du Canal de Provence pour la qualité des moyens de travail qui nous a été offerte pendant l'expérimentation. 


\section{ANNEXES}

\section{Unités}

$\mathrm{ml}$ mètre linéaire

$\mathrm{m}^{3} \cdot \mathrm{s}^{-1} \cdot \mathrm{ml}^{-1}$ débit par unité de longueur de seuil $\mathrm{m} / \mathrm{m} \quad$ pente du déversoir

\section{Notations}

$Y_{c} \quad$ Tirant d'eau critique

$Y_{1} \quad$ Tirant d'eau au pied du déversoir

$Y_{2}$ Tirant d'eau conjugué à $Y_{1}$ en aval du ressaut
$Y_{p} \quad$ Tirant d'eau du coussin d'eau sous la lame déversante

$E_{0}$ Charge totale de l'écoulement sur le seuil du déversoir

$E_{1}$ Charge totale de l'écoulement au pied du déversoir

$q \quad$ Débit par unité de longueur de seuil

$g$ Accélération de la gravité

$H$ Hauteur du déversoir

$h \quad$ Hauteur d'un gradin

$n \quad$ Nombre de gradins du déversoir

$i \quad$ Pente du déversoir

$V_{m}$ Vitesse de l'écoulement dans la section $m$

$H_{s}$ Charge spécifique : $H_{s}=Y+V^{2} / 2 g$

\section{Terminologie relative aux gradins}

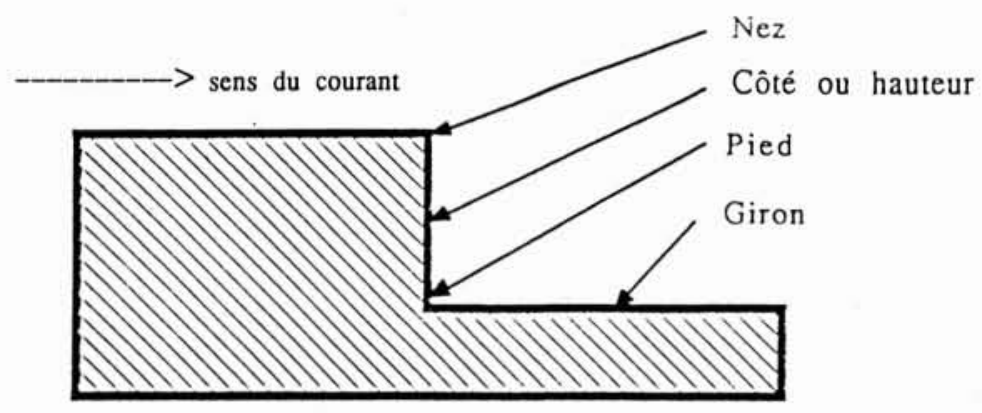

\section{Bibliographie}

[1] Agostini (R.), Bizzari (A.), Masetti (M.): Ouvrages flexibles pour tronçons torrentiels et fluviaux ( $I^{\text {re }}$ partie: Ouvrages transversaux). Ed. France Gabions s.a., Le Pouzin, 1987.

[2] CEMAGREF : Les ouvrages en gabions. En cours d'édition par le Ministère Français de la Coopération et du Développement (Edition précédente: Collection Techniques Rurales en Afrique, Ed. Eyrolles, Paris, 1974).

[3] Durand (J.-M.): Etude de variantes techniques dans la conception des petits barrages en terre au Burkina Faso. Ed. CEMAGREF groupement d'Aix-en-Provence, Le Tholonet, mai 1989.

[4] ESSERY (S.), HORNER (W.): The hydraulic design of stepped spillways (CIRIA Report 33). Ed. CIRIA (Construction Industry Research and Information Association), Londres, janvier 1978.

[5] Peyras (L.): Etude de la dissipation de l'énergie sur les déversoirs en gradins de gabions. Ed. CEMAGREF groupement d'Aix- en-Provence, Le Tholonnet, mai 1990.
[6] Rajaratnam (N.) : Skimming flow in stepped spillways. $J$. hydraul. eng., $A S C E$, vol. 116, $\mathrm{n}^{\circ} 4$, avril 1990, pp. 587 . 591.

[7] RAND (W.): Flow geometry at straight drop spillways. Proc. ASCE, 81 E, Paper 791, septembre 1955, pp. 1-13.

[8] Sorensen (R. M.): Stepped spillway hydraulic mode investigation. J. hydraul. eng., ASCE, vol. 111, $\mathrm{n}^{*} 12$, décembre 1985, pp. 1461-1472.

[9] Stephenson (D.): Gabion energy dissipators. Treizième congrès des grands barrages, New-Delhi, 1979. Ed. Commission Internationale des Grands Barrages, Q. 50, R. 3 , pp. 33-43, 1979.

[10] STEPHENSON (D.): Rockfill in hydraulic engineering. Ed. Elsevier scientific publishing company, Collection Developments in geotechnical engineering, vol. 27, Amsterdam, Oxford, New York, 1979.

[11] United States Department OF the INTERIOR (Bureau of Reclamation). Design of small dams. Ed. Water Resources Technical Publication (United States Government Printing Office), Denver, 1987. 